\title{
Efeitos da plataforma vibratória no equilíbrio, atividade eletromiográfica e propriocepção de idosos
}

Effects of the vibratory platform on balance, electromyographic activity and proprioception of the elderly

Efectos de la plataforma vibratoria sobre el equilibrio, la actividad electromiográfica y la propiocepción de los ancianos

\section{Resumo}

Este estudo teve como objetivo comparar os efeitos de um treinamento sensório-motor em idosos, sobre uma plataforma vibratória e no solo. Participaram 3 idosos, de ambos os gêneros, entre 61 e 66 anos. O paciente 1 realizou treinamento sensório-motor sobre a plataforma vibratória e os pacientes 2 e 3 no solo. Eles foram avaliados antes, após um dia e após cinco dias de tratamento. Os pacientes 1 e 2 tiveram melhora do equilíbrio após um dia de tratamento. Após cinco dias, somente a paciente 1 apresentou melhora. A paciente 3 teve melhora após um dia de tratamento, que se manteve após os cinco dias. A paciente 1 apresentou aumento da atividade elétrica de todos os músculos avaliados. A paciente 2 apresentou aumento da atividade elétrica dos músculos fibular longo, vasto medial e glúteo médio. O músculo gastrocnêmio medial apresentou aumento somente após os cinco dias. Houve aumento da atividade elétrica do músculo vasto medial da paciente 3 em todas as avaliações. O glúteo médio apresentou aumento somente após cinco dias. No ângulo de $60^{\circ}$ de extensão do joelho, os pacientes 1 e 2 apresentaram melhora da propriocepção somente após os cinco dias. No ângulo de $30^{\circ}$, a paciente 1 teve melhora após um dia e após os cinco dias, enquanto o paciente 2 apresentou melhora somente após o fim do tratamento. A paciente 3 teve melhora em todas as avaliações para $60^{\circ}$ e $30^{\circ}$ de extensão do joelho. Verificou-se que ambos os tratamentos foram benéficos para os idosos.

Palavras-chave: Envelhecimento; Equilíbrio postural; Propriocepção; Acidentes por quedas; Vibração.

\section{Abstract}

This study aimed to compare the effects of a physical-motor training in the elderly, on a vibratory platform and on the ground. Participants were three elderly, of both genders, between 61 and 66 years old. Patient 1 underwent sensortorymotor training on the vibratory platform and patients 2 and 3 on the ground. They were evaluated before, after one day and after five days of treatment. Patients 1 and 2 had improved balance after one day of treatment. After five days, only patient 1 showed improvement. Patient 3 improved after one day of treatment, which was maintained after 
five days. Patient 1 showed increased electrical activity of all muscles evaluated. Patient 2 showed increased electrical activity of the long fibular, vastus medial and gluteus media. The medial gastrocnemius muscle increased only after five days. There was an increase in the electrical activity of the vastus medial muscle of patient 3 in all evaluations. The middle gluteus increased only after five days. At the $60^{\circ}$ angle of knee extension, patients 1 and 2 showed improvement of proprioception only after five days. No ângulo de $30^{\circ}$, a paciente 1 teve melhora após um dia e após os cinco dias, enquanto o paciente 2 apresentou melhora somente após o fim do tratamento. A paciente 3 teve melhora em todas as avaliações para $60^{\circ}$ e $30^{\circ}$ de extensão do joelho. Verificou-se que ambos os tratamentos foram benéficos para os idosos.

Keywords: Aging; Postural balance; Proprioception; Accidents by falls; Vibration.

\section{Resumen}

Este estudio tuvo como objetivo comparar los efectos de un entrenamiento físico-motor en los ancianos, en una plataforma vibratoria y en el suelo. Los participantes fueron tres ancianos, de ambos sexos, entre 61 y 66 años. El paciente 1 se sometió a entrenamiento sensortorio-motor en la plataforma vibratoria y los pacientes 2 y 3 en el suelo. Fueron evaluados antes, después de un día y después de cinco días de tratamiento. Los pacientes 1 y 2 habían mejorado el equilibrio después de un día de tratamiento. Después de cinco días, solo el paciente 1 mostró mejoría. El paciente 3 mejoró después de un día de tratamiento, que se mantuvo después de cinco días. El paciente 1 mostró un aumento de la actividad eléctrica de todos los músculos evaluados. El paciente 2 mostró un aumento de la actividad eléctrica del fibular largo, vasto medial y glúteo medio. El músculo gastrocnemio medial aumentó solo después de cinco días. Hubo un aumento en la actividad eléctrica del músculo vasto medial del paciente 3 en todas las evaluaciones. El glúteo medio aumentó solo después de cinco días. En el ángulo de $60^{\circ}$ de extensión de la rodilla, los pacientes 1 y 2 mostraron mejoría de la propiocepción solo después de cinco días. En el ángulo de $30^{\circ}$, el paciente 1 mejoró después de un día y después de cinco días, mientras que el paciente 2 mostró mejoría solo después del final del tratamiento. El paciente 3 mejoró en todas las evaluaciones para la extensión de rodilla de $60^{\circ}$ y $30^{\circ}$. Se encontró que ambos tratamientos fueron beneficiosos para los ancianos.

Palabras clave: Envejecimiento; Equilibrio postural; Propiocepción; Accidentes por caídas; Vibración.

\section{Introdução}

O aumento da expectativa de vida e a queda da taxa de natalidade ocasionaram um intenso envelhecimento na população mundial. Todo ano, 700 mil novos idosos são incorporados à população brasileira (Veras, 2016). De acordo com o Instituto Brasileiro de Geografia e Estatística (IBGE) (2018), o país ultrapassou a marca de 30 milhões de idosos em 2017. Isso tem gerado desafios econômicos e sociais, além de aumentar a necessidade de pesquisas na área do envelhecimento (Júnior et al., 2019).

O avanço da idade é, geralmente, acompanhado pelo declínio das funções biológicas, físico-motoras e cognitivas, com maior incidência de agravos à saúde, sendo assim, ainda que sem doenças crônicas, o processo de envelhecimento envolve perdas funcionais, exigindo que o cuidado com o idoso seja idealizado de maneira diferente do que é realizado com adultos jovens (Hayter et al., 2015; Veras \& Oliveira, 2018).

O envelhecimento naturalmente ocasiona perda de trofismo e força muscular, assim como diminuição da propriocepção, ou seja, redução da capacidade de percepção do movimento articular (Varella et al., 2016; Gusmão \& Reis, 2017; Sumukadas, 2018). Essas modificações podem levar à instabilidade postural, causa mais comum de quedas em idosos (Richardson, 2017).

A queda é o deslocamento não intencional do corpo para um nível inferior, com incapacidade de correção do indivíduo em tempo hábil, podendo causar complicações como limitações funcionais e perda da independência (Agudelo-Botero et al., 2018). Ela é a segunda principal causa de mortes acidentais no mundo, sendo que a mortalidade é maior entre os idosos (Organização Mundial da Saúde, 2018). No Brasil, estudo realizado em 70 municípios mostrou que a prevalência de quedas na população idosa foi de 25,1\% (Pimentel et al., 2018).

Alguns protocolos de exercícios podem ser usados para melhora do equilíbrio em idosos, visando à prevenção de quedas. O treinamento sensório-motor é um dos recursos utilizados por fisioterapeutas para aumentar as habilidades de controle postural, restaurando a estabilidade estática e dinâmica do indivíduo (Ferlinc et al., 2019). Novas modalidades deste 
treinamento mostram-se promissoras, como a Vibração de Corpo Inteiro (VCI), que consiste em ficar sobre uma plataforma que oscila em uma determinada freqüência e amplitude, ativando contrações musculares através da estimulação dos receptores sensoriais (Rogan et al., 2017).

Embora a literatura aponte os benefícios da VCI para a população idosa, ainda faltam conhecimentos e evidências mais aprofundadas. Estudos envolvendo idosos mostram a heterogeneidade das metodologias empregadas e as divergências com relação à resposta do organismo aos estímulos vibratórios, dificultando a tomada de decisão clínica por parte dos fisioterapeutas (Orr, 2015; Bacha et al., 2016; Pessoa et al., 2017; Rogan et al., 2017; Fischer et al., 2019).

Sendo assim, o objetivo deste estudo foi comparar os efeitos de um treinamento sensório-motor em idosos, sobre uma plataforma vibratória e em superfície estável.

\section{Metodologia}

Este estudo foi aprovado pelo Comitê de Ética em Pesquisa (CEP) da Universidade do Vale do Paraíba (UNIVAP), sob o CAAE 18643413.3.0000.5503 (ANEXO A), respeitando todos os princípios éticos que norteiam pesquisas envolvendo seres humanos, bem como a privacidade dos seus conteúdos, como preconiza a Resolução 466/12 do Conselho Nacional de Saúde do Ministério da Saúde. O estudo encontra-se registrado no Clinical Trials sob o número: NCT03846037.

Por meio do Termo de Consentimento Livre e Esclarecido (TCLE), os participantes foram previamente esclarecidos sobre os objetivos do estudo, riscos, benefícios e os procedimentos aos quais seriam submetidos. A aceitação plena foi confirmada após a assinatura do TCLE, permanecendo uma via com o participante e outra com a pesquisadora responsável.

Trata-se de um estudo de três casos, realizado nas dependências do Laboratório de Engenharia de Reabilitação Sensório Motora (LERSM), pertencente ao Instituto de Pesquisa \& Desenvolvimento (IP\&D) e dos Laboratórios de Biodinâmica e de Reabilitação Cardiopulmonar, pertencentes à Faculdade de Ciências da Saúde da UNIVAP.

Participaram deste estudo 3 idosos, de ambos os gêneros, na faixa etária entre 61 e 66 anos, residentes na comunidade, em um município do interior do estado de São Paulo, respeitando os critérios de elegibilidade para o estudo.

Foram incluídos idosos sedentários; com uma adequada compreensão e competências de comunicação; que não estivessem sob outro tratamento fisioterapêutico e que assinaram o TCLE. Com base no que é preconizado pela Organização Mundial da Saúde (OMS) (2020) em novas diretrizes globais sobre atividade física e comportamento sedentário, considerou-se sedentário o indivíduo que não praticasse no mínimo 150 minutos de atividade física intensa semanal, em casos em que não houvesse contraindicações.

Os critérios de exclusão foram: idosos submetidos a qualquer tipo de intervenção cirúrgica há menos de 6 meses; portadores de marca-passo; que apresentassem doenças que interferem no equilíbrio; doenças cardiovasculares não controladas; lesões musculoesqueléticas, implantes metálicos e/ou dor nos membros inferiores; com déficits visuais e/ou auditivos não corrigidos; que estivessem em uso de medicamentos que alteram o equilíbrio; que ingerissem bebida alcoólica antes das sessões de tratamento, além daqueles que relatassem desconforto durante o protocolo de avaliação ou intervenção e que interrompessem o tratamento.

Os participantes foram recrutados, avaliados e após distribuição aleatória por sorteio, designados para o treinamento sensório-motor sobre a plataforma vibratória ou em superfície estável (Fluxograma 1). A designação para cada tratamento foi realizada por um pesquisador independente que não estava envolvido no recrutamento e/ou na intervenção. 
Figura 1 - Fluxograma com as etapas sequenciais dos procedimentos da pesquisa.

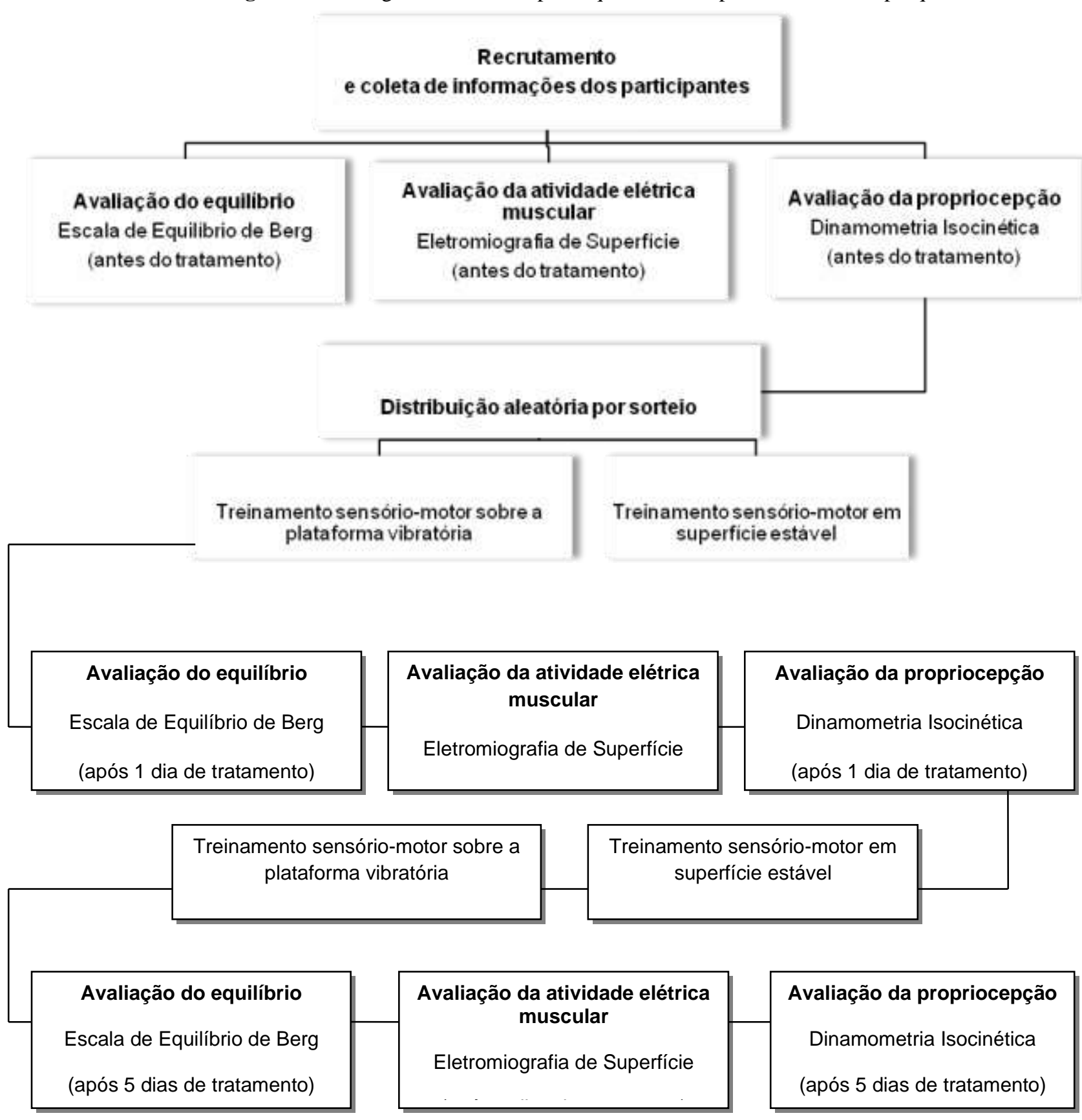

Fonte: Autores.

Foram coletadas informações referentes a gênero; idade; doenças crônicas; histórico de quedas; massa corporal; altura; Índice de Massa Corporal (IMC), dado pelo quociente entre a massa corporal (expressa em quilograma - Kg) pela altura ao quadrado (expressa em metros - m) e membro inferior não dominante dos participantes.

O domínio do membro inferior foi definido como a perna preferida para chutar uma bola, já que em um artigo de revisão realizado por McGrath et al. (2016), essa definição mostrou-se mais eficaz na determinação das diferenças entre os membros inferiores no controle postural unipodal. O membro inferior dominante pode apresentar melhor desempenho na realização de tarefas, quando comparado ao membro não dominante. O desequilíbrio muscular entre os lados pode causar sobrecarga e compensações que alteram o movimento (Queiroz et al., 2019), isso demonstra a importância de se avaliar e treinar o membro inferior não dominante, sobretudo dos idosos. 
Todos os participantes foram avaliados antes e após um dia de tratamento e após cinco sessões consecutivas do treinamento sensório-motor. Eles foram orientados a não praticar exercício físico neste período.

Para avaliar o equilíbrio dos participantes, foi utilizada a Berg Balance Scale, criada por Berg et al. (1992), sendo traduzida, adaptada e validada à população brasileira por Miyamoto et al. (2004). A literatura aponta uma variedade de testes com o objetivo de avaliar o equilíbrio e estabelecer parâmetros para a identificação de idosos com maior risco de sofrerem quedas. Porém, autores destacam a vantagem da EEB com relação aos demais instrumentos, já que, necessitando de poucos equipamentos para ser administrada, ela avalia aspectos diferentes do equilíbrio, além de verificar a eficácia de intervenções na prática clínica e científica (Berg et al., 1992; Miyamoto et al., 2004; Diego et al., 2012; Neto \& Brito, 2015).

A escala é considerada segura para a avaliação do equilíbrio estático e dinâmico de idosos baseada em 14 tarefas representativas de atividades da vida diária tais como: levantar-se; permanecer em pé e sentado sem apoio; sentar-se; transferirse de uma cadeira com apoio para uma sem apoio; permanecer em pé com os olhos fechados sem apoio e com os pés juntos; inclinar-se para frente; pegar um objeto do chão; virar-se para trás; girar $360^{\circ}$; posicionar os pés alternadamente em um degrau; permanecer em pé sem apoio com um pé à frente do outro e sobre uma perna só (Miyamoto et al., 2004).

Os testes avaliam tanto a forma como é realizada cada tarefa, como o tempo para realizá-las. Cada item possui cinco alternativas que variam de 0 a 4 pontos. A capacidade em realizar a atividade de modo independente é expressa na escala através da pontuação 4; quando há necessidade de supervisão por parte do avaliador a pontuação é 3; quando o indivíduo necessita de segurança e auxílio moderado a pontuação é 2; quando há necessidade de auxílio e o tempo de permanência na postura de equilíbrio é mínima a pontuação é 1 e se o indivíduo for incapaz de realizar a tarefa de maneira independente a pontuação é 0 . O score total varia de 0 a 56 pontos, sendo que quanto maior for à pontuação, melhor é o equilíbrio e menor é o risco de quedas (Berg, 1992).

Os materiais utilizados para a aplicação da escala foram: duas cadeiras (uma com braços e outra sem); um cronômetro; um banquinho; uma régua e uma escada auxiliar de consultório. Para cada tarefa, foram dadas instruções por meio de comando verbal e a atividade era demonstrada para o voluntário. O tempo de realização da avaliação foi de aproximadamente 20 minutos.

A Eletromiografia de Superfície (EMGS) tem sido amplamente utilizada para se compreender as funções e disfunções do sistema muscular. Este é um recurso não invasivo que permite o registro dos sinais mioelétricos gerados pelas células musculares, possibilitando a análise da atividade muscular por fisioterapeutas, para avaliar efeitos de intervenções terapêuticas, dentre outros objetivos (Schmidt et al., 2020).

Para a aquisição dos dados eletromiográficos, foi utilizado o Eletromiógrafo de 8 canais (EMG System do Brasil 830), portátil, com Wi-Fi, calibrado com as seguintes especificações técnicas: Ymin: 0; Ymáx: 200; Coef.A: 218,85 e Coef.B: 120,31, com placa de conversão A/D (conversor analógico-digital) com 12 bits de resolução e eletrodo bipolar pré-amplificado com um ganho diferencial de 20 vezes, totalizando uma amplificação final de 1000 vezes.

Foram utilizados eletrodos reutilizáveis de prata/cloreto de prata $(\mathrm{Ag} / \mathrm{AgCl})$ em forma de disco, com borda plástica e centro de metal, com diâmetro de contato de $10 \mathrm{~mm}$, fixados aos pares entre o ponto motor e o tendão distal de cada músculo a ser avaliado, com uma distância de $20 \mathrm{~mm}$ entre eles. Antes da colocação dos eletrodos realizou-se a tricotomia, quando necessária, e a higienização da região com algodão embebido em álcool a 70\%, com o intuito de diminuir a impedância da pele. Os eletrodos foram acoplados à pele por gel condutor e fixados com discos adesivos descartáveis e fita adesiva.

Os eletrodos foram posicionados aos pares entre o ponto motor e o tendão distal dos músculos fibular longo, gastrocnêmio medial, vasto medial e glúteo médio, do membro inferior não dominante dos participantes, conforme as recomendações do Surface Electromyography for the Non Invasive Assessment of Muscle (SENIAM). Este é um projeto 
desenvolvido no âmbito do Programa de Investigação e Saúde Biomédica (BIOMED II) da União Europeia, a fim de integrar a investigação e aplicação da EMGS.

A avaliação do músculo fibular longo foi feita da seguinte maneira: com o participante sentado e o membro inferior girado medialmente, os eletrodos foram colocados a $25 \%$ acima da linha entre a cabeça da fíbula e o maléolo lateral. Orientouse que o indivíduo apoiasse o membro inferior acima da articulação do tornozelo, invertesse o pé com flexão plantar enquanto aplica pressão contra a borda lateral e a sola do pé, na direção de inversão do pé e dorsiflexão do tornozelo.

Com o participante em decúbito ventral, o joelho estendido e o pé projetando-se sobre o final da maca, os eletrodos foram posicionados no ponto mais proeminente do músculo gastrocnêmio medial, na direção da perna. Foi orientado que o indivíduo realizasse uma flexão plantar da articulação do tornozelo.

A avaliação do músculo vasto medial foi feita com o participante sentado na maca, os joelhos em leve flexão e a parte superior do corpo ligeiramente inclinada para trás, os eletrodos foram posicionados a $80 \%$ da linha entre a espinha ilíaca ântero-superior e o espaço articular do joelho na frente do ligamento colateral medial, com os eletrodos perpendiculares a esta linha. Foi orientado que o indivíduo estendesse o joelho sem girar a coxa enquanto aplica pressão contra a perna acima do tornozelo na direção da flexão.

Com o participante em decúbito lateral, foi avaliado o músculo glúteo médio. Os eletrodos foram posicionados no ponto médio da linha entre a crista ilíaca e o trocânter maior do fêmur, com os eletrodos na direção desta linha. Foi orientado que o indivíduo realizasse abdução do quadril contra resistência manual.

Todos os registros foram realizados com os voluntários em apoio unipodal por 20 segundos sobre o membro inferior não dominante. $\mathrm{O}$ eletrodo de referência do tipo pregador, revestido com gel condutor, foi posicionado sobre o processo estilóide da ulna no lado contralateral ao membro inferior avaliado. Para a análise dos dados, considerando o tempo total da coleta, foi selecionado apenas o trecho entre os dois segundos iniciais e finais, a fim de diminuir os efeitos de adaptação e relaxamento.

Para avaliação da propriocepção foi utilizado o Dinamômetro Isocinético Computadorizado Biodex System 3 (EMG System do Brasil) acionado no modo Propriocepton. Este equipamento contém programações específicas para avaliação da percepção da posição articular. Assim, a propriocepção é avaliada pelo erro dessa percepção em relação a uma posição prédefinida. Havendo redução deste erro, pode-se afirmar que houve melhora da propriocepção da articulação avaliada (Thomaz, 2015).

A avaliação proprioceptiva foi realizada na articulação do joelho do membro inferior não dominante dos participantes. A articulação do joelho tem um papel substancial no equilíbrio humano e a integridade de sua propriocepção é essencial para o controle neuromotor da articulação. Sendo assim, sua avaliação é um importante método de informação diagnóstica e prognóstica, podendo gerar evidência da eficácia ou não de intervenções terapêuticas (Kilby et al., 2015).

Os participantes foram colocados na cadeira do dinamômetro e estabilizados na posição sentada por cintos na região do tronco, cruzando a região da pelve e coxa e ao redor do tornozelo, com a fossa poplítea a pelo menos $10 \mathrm{~cm}$ fora do assento da cadeira, a fim de eliminar possíveis interferências cutâneas. A articulação do joelho foi posicionada inicialmente a $90^{\circ}$ de flexão e o eixo articular foi alinhado com o eixo do dinamômetro.

A articulação do joelho dos participantes foi lentamente estendida pelo examinador, junto ao equipamento, a uma velocidade angular de $20^{\circ}$ por segundo, partindo da angulação de $90^{\circ}$ de flexão, até as angulações de extensão prédeterminadas: $60^{\circ}$ e $30^{\circ}$. Já posicionados na cadeira do dinamômetro e com a oclusão da visão, os voluntários foram instruídos a memorizar o ângulo, no tempo de 10 segundos, e depois retornar para a angulação de $90^{\circ}$ de flexão do joelho. Em seguida, foi solicitada aos voluntários a extensão ativa do joelho até o momento em que percebessem o ângulo-alvo testado, interrompendo o movimento por meio de um disparador manual. Cada voluntário realizou três tentativas para cada ângulo- 
alvo. Todos os voluntários desconheciam o valor numérico dos ângulos-alvo utilizados e para que aprendessem a realizar o teste no equipamento, eles executaram um pré-teste com angulações diferentes.

Após as avaliações iniciais, os participantes realizaram o mesmo protocolo de treinamento sensório-motor, mas em superfícies diferentes. Os voluntários foram submetidos ao treinamento em um período de 5 dias consecutivos, com uma sessão para cada dia. Em pé, descalços, com o tronco ereto, cabeça na posição neutra, olhar fixo na linha do horizonte e membros superiores abduzidos a $90^{\circ}$, os participantes foram orientados a sustentar a postura de apoio unipodal sobre o membro inferior não dominante, com o joelho contralateral fletido a $90^{\circ}$, em 4 séries durante 60 segundos intervaladas por 60 segundos de repouso em ortostase. $\mathrm{O}$ treinamento em apoio unipodal exige mais de todas as articulações e musculaturas do membro inferior, sendo amplamente utilizado em estudos (Donath, 2015; Rodrigues \& Bertoncello, 2017).

Para a realização da VCI foi utilizada uma Plataforma Vibratória triplanar (Vibra, Nissan Fisio®) no modo de vibração contínua, com uma amplitude de movimento orbital de $6 \mathrm{~mm}$ e frequência de vibração ajustada em $30 \mathrm{~Hz}$ (Figura 15). De acordo com Santos et al., (2019), frequências entre 20 a $40 \mathrm{~Hz}$ parecem apresentar bons resultados para os idosos. O treinamento sensório-motor em superfície estável foi realizado em um solo plano com piso aderente. Todos os participantes foram acompanhados pela pesquisadora que ofereceu ajuda somente no caso de desequilíbrio com risco de queda, situação na qual foi fornecido apoio aos membros superiores e suporte ao corpo.

Os dados coletados no estudo foram tabulados no Microsoft Office Excel $2010^{\circledR}$. Para análise dos resultados da EEB foi calculado o percentual de diferença entre as pontuações obtidas pelos participantes nas avaliações. A pontuação total consiste na soma de cada item verificado pelo examinador ao aplicar a escala. Os dados provenientes da EMGS e da dinamometria isocinética foram analisados e interpretados pelos softwares próprios de cada equipamento.

Para a análise dos sinais e cálculo do Root Mean Square (RMS), expressa em V, foi utilizado o programa EMGWorks 4.0 Analysis: utilizou-se o filtro Butterworth de $4^{\circ}$ ordem, como limite inferior $10 \mathrm{~Hz}$ e limite superior de $500 \mathrm{~Hz}$, frequência de amostragem definida para $2 \mathrm{kHz}$ por canal. Em seguida, foi calculado o percentual de diferença entre os valores de RMS obtidos nas avaliações.

Quanto à análise da dinamometria isocinética, foi considerado o ângulo de erro, resultado da média aritmética das diferenças entre os ângulos determinados pelo examinador e a angulação alcançada pelos participantes em cada uma das tentativas. Posteriormente, foi calculado o percentual de diferença entre as médias dos erros.

\section{Resultados}

Todos os participantes completaram o protocolo do estudo. Um deles realizou o treinamento sensório-motor sobre a plataforma vibratória e os outros dois em superfície estável.

Paciente 1, gênero feminino, 61 anos, sedentária, nega doenças crônicas, sem histórico de quedas, massa corporal de $55 \mathrm{~kg}$, com 1,58m de altura, IMC de 22,03 e membro inferior direito dominante. Após distribuição aleatória por sorteio, a paciente realizou o treinamento sensório-motor sobre a plataforma vibratória.

A pontuação (score) obtida pela participante 1 na EEB está representada na Tabela 1, com o cálculo do percentual de diferença entre a avaliação basal vs $1^{\circ}$ dia; avaliação basal vs $5^{\circ}$ dia e avaliação $1^{\circ}$ dia vs $5^{\circ}$ dia. Os resultados mostram que, houve melhora do desempenho na EEB, tendo em vista o aumento dos scores obtidos pela participante após um dia de tratamento e após os cinco dias de intervenção. 
Tabela 1 - Comparação das pontuações obtidas pela participante 1 na EEB nas três avaliações.

\begin{tabular}{lcccc}
\hline Avaliações & Score & \% Basal vs $\mathbf{1}^{\mathbf{0}}$ dia & \% Basal vs $\mathbf{5}^{\mathbf{0}}$ dia & $\mathbf{\%}^{\mathbf{0}}$ dia vs $\mathbf{5}^{\circ}$ dia \\
\hline Avaliação basal & 51 & $\uparrow 5,88$ & $\uparrow 7,84$ & $\uparrow 1,85$ \\
$1^{\text {o }}$ sessão de TTO & 54 & - & - & - \\
$5^{\circ}$ sessão de TTO & 55 & - & - & - \\
\hline
\end{tabular}

$\mathrm{TTO}=$ Tratamento; $\%=$ Porcentagem; $\uparrow=$ Aumento. Fonte: Autores.

Na Tabela 2, estão descritos os resultados referentes ao valor de RMS dos músculos avaliados pela EMGS da participante 1 , com o percentual de diferença entre a avaliação basal vs $1^{\circ}$ dia; avaliação basal vs $5^{\circ}$ dia e avaliação $1^{\circ}$ dia vs $5^{\circ}$ dia. Quando comparadas as três avaliações, os dados mostram que houve aumento do RMS de todos os músculos avaliados, com destaque para o músculo fibular longo, que apresentou aumento expressivo da atividade elétrica.

Tabela 2 - Análise da EMGS dos músculos fibular longo, gastrocnêmio medial, vasto medial e glúteo médio da participante 1 nas três avaliações.

\begin{tabular}{ccccccc}
\hline $\begin{array}{c}\text { Músculo Valor } \\
\text { RMS (V) }\end{array}$ & $\begin{array}{c}\text { Avaliação } \\
\text { basal }\end{array}$ & $\begin{array}{c}\mathbf{1}^{\mathbf{o}} \text { sessão de } \\
\text { TTO }\end{array}$ & $\mathbf{5}^{\mathbf{0}}$ sessão de TTO & $\begin{array}{c}\text { \% Basal } \\
\text { vs } \\
\mathbf{1}^{\mathbf{0}} \text { dia }\end{array}$ & $\begin{array}{c}\text { \% Basal } \\
\text { vs } \\
\mathbf{5}^{\mathbf{0}} \text { dia }\end{array}$ & $\begin{array}{c}\text { \% } \mathbf{1}^{\mathbf{0}} \text { dia } \\
\text { vs } \\
\mathbf{5}^{\mathbf{0}} \text { dia }\end{array}$ \\
\hline $\begin{array}{c}\text { Fibular longo } \\
\text { Gastrocnêmio } \\
\text { medial }\end{array}$ & 22,4 & 22,86 & 223,84 & $\uparrow 2,05$ & $\uparrow 999,28$ & $\uparrow 979,18$ \\
Vasto medial & 10,51 & 71,71 & 84,09 & $\uparrow 1,70$ & $\uparrow 19,26$ & $\uparrow 17,26$ \\
Glúteo médio & 11,76 & 14,93 & 17,26 & $\uparrow 26,95$ & $\uparrow 46,77$ & $\uparrow 15,61$ \\
\hline
\end{tabular}

$\mathrm{V}=$ Volt; TTO $=$ Tratamento; $\%=$ Porcentagem; $\uparrow=$ Aumento. Fonte: Autores.

Na Tabela 3 são apresentadas as médias dos erros referentes à propriocepção em $60^{\circ}$ e $30^{\circ}$ de extensão do joelho avaliadas pela dinamometria isocinética da participante 1 , com o percentual de diferença entre a avaliação basal vs $1^{\circ}$ dia; avaliação basal vs $5^{\circ}$ dia e avaliação $1^{\circ}$ dia vs $5^{\circ}$ dia. As médias das três tentativas seguidas mostram que houve melhora do senso de posição articular do joelho comparando os períodos após um dia de tratamento e após as cinco sessões para o ângulo de $60^{\circ}$ de extensão, tendo em vista a redução dos erros. Para os $30^{\circ}$ de extensão a melhora do senso de posição articular se mostrou mais significativa, já que a redução dos erros foi verificada ao comparar as três avaliações.

Tabela 3 - Médias dos erros nas três avaliações da propriocepção da participante 1 , nas angulações de $60^{\circ}$ e $30^{\circ}$ de extensão de joelho no dinamômetro isocinético.

\begin{tabular}{|c|c|c|c|c|c|c|c|c|}
\hline \multirow[b]{2}{*}{ Avaliações } & \multicolumn{2}{|c|}{ Angulações } & \multicolumn{3}{|c|}{$60^{\circ}$} & \multicolumn{3}{|c|}{$\mathbf{3 0}^{\circ}$} \\
\hline & $60^{\circ}$ & $30^{\circ}$ & $\begin{array}{c}\text { \% Basal } \\
\text { vs } \\
1^{\circ} \text { dia }\end{array}$ & $\begin{array}{c}\% \text { Basal } \\
\text { vs } \\
5^{\circ} \text { dia }\end{array}$ & $\begin{array}{c}\% 1^{\circ} \text { dia vs } \\
5^{\circ} \text { dia }\end{array}$ & $\begin{array}{c}\text { \% Basal } \\
\text { vs } \\
1^{\circ} \text { dia }\end{array}$ & $\begin{array}{c}\% \text { Basal vs } \\
5^{\circ} \text { dia }\end{array}$ & $\begin{array}{c}\% 1^{\circ} \text { dia vs } \\
5^{\circ} \text { dia }\end{array}$ \\
\hline Avaliação basal & 4 & 6.7 & $\uparrow 25$ & $\uparrow 7,5$ & $\downarrow 14$ & $\downarrow 10,44$ & $\downarrow 14,92$ & $\downarrow 5$ \\
\hline $\begin{array}{c}1^{\circ} \text { sessão de } \\
\text { TTO }\end{array}$ & 5 & 6 & - & - & - & - & - & - \\
\hline $\begin{array}{c}5^{\circ} \text { sessão de } \\
\text { TTO }\end{array}$ & 4.3 & 5.7 & - & - & - & - & - & - \\
\hline
\end{tabular}

$\mathrm{TTO}=$ Tratamento; \%= Porcentagem; $\uparrow=$ Aumento; $\downarrow$ Diminuição. Fonte: Autores. 
Paciente 2, gênero masculino, 66 anos, sedentário, diabético em uso de medicação para controle, sem histórico de quedas, massa corporal de $80 \mathrm{~kg}$, com 1,70m de altura, IMC de 27,68 e membro inferior direito dominante. Após distribuição aleatória por sorteio, o paciente realizou o treinamento sensório-motor em superfície estável.

A pontuação (score) obtida pelo participante 2 na EEB está representada na Tabela 4, com o cálculo do percentual de diferença entre a avaliação basal vs $1^{\circ}$ dia; avaliação basal vs $5^{\circ}$ dia e avaliação $1^{\circ}$ dia vs $5^{\circ}$ dia. Os resultados mostram que, houve melhora do desempenho na EEB, tendo em vista o aumento do score obtido pelo participante após um dia de tratamento, que se manteve após os cinco dias de intervenção.

Tabela 4 - Comparação das pontuações obtidas pelo participante 2 na EEB nas três avaliações.

\begin{tabular}{|c|c|c|c|c|}
\hline Avaliações & Score & $\%$ Basal vs $1^{\circ}$ dia & $\%$ Basal vs $5^{\circ}$ dia & $\% 1^{\circ}$ dia vs $5^{\circ}$ dia \\
\hline Avaliação basal & 52 & $\uparrow 3,85$ & $\uparrow 3,85$ & 0 \\
\hline $1^{\circ}$ sessão de TTO & 54 & - & - & - \\
\hline $5^{\circ}$ sessão de TTO & 54 & - & - & - \\
\hline
\end{tabular}

$\mathrm{TTO}=$ Tratamento; $\%=$ Porcentagem; $\uparrow=$ Aumento. Fonte: Autores.

Na Tabela 5, estão descritos os resultados referentes ao valor de RMS dos músculos avaliados pela EMGS do participante 2 , com o percentual de diferença entre a avaliação basal vs $1^{\circ}$ dia; avaliação basal vs $5^{\circ}$ dia e avaliação $1^{\circ}$ dia vs $5^{\circ}$ dia. Observou-se que houve aumento do RMS comparando as três avaliações dos músculos fibular longo, vasto medial e glúteo medial, sendo estes dois últimos os que mais se destacaram em relação aos valores do RMS e diferenças percentuais. O músculo gastrocnêmio medial apresentou redução da atividade elétrica após um dia de tratamento e aumento após os cinco dias de intervenção.

Tabela 5 - Análise da EMGS dos músculos fibular longo, gastrocnêmio medial, vasto medial e glúteo médio do participante 2.

\begin{tabular}{|c|c|c|c|c|c|c|}
\hline $\begin{array}{c}\text { Músculo } \\
\text { Valor RMS } \\
\text { (V) }\end{array}$ & $\begin{array}{l}\text { Avaliação } \\
\text { basal }\end{array}$ & $\begin{array}{c}1^{\circ} \text { sessão de } \\
\text { TTO }\end{array}$ & $5^{\circ}$ sessão de TTO & $\begin{array}{c}\% \text { Basal } \\
\text { vs } \\
1^{\circ} \text { dia }\end{array}$ & $\begin{array}{c}\% \text { Basal } \\
\text { vs } \\
5^{\circ} \text { dia }\end{array}$ & $\begin{array}{c}\% 1^{0} \text { dia } \\
\text { vs } \\
5^{\circ} \text { dia }\end{array}$ \\
\hline Fibular longo & 219,86 & 220,46 & 223,53 & $\uparrow 0,27$ & $\uparrow 1,67$ & $\uparrow 1,39$ \\
\hline Gastrocnêmio medial & 99,77 & 67,58 & 84,18 & $\downarrow 32,26$ & $\downarrow 15,63$ & $\uparrow 24,56$ \\
\hline Vasto medial & 5,46 & 12,69 & 17,25 & $\uparrow 232,42$ & $\uparrow 135,93$ & $\uparrow 35,93$ \\
\hline Glúteo médio & 7,71 & 17,98 & 22,23 & $\uparrow 133,20$ & $\uparrow 188,33$ & $\uparrow 23,64$ \\
\hline
\end{tabular}

$\mathrm{V}=$ Volt; TTO= Tratamento; \%= Porcentagem; $\uparrow=$ Aumento; $\downarrow=$ Diminuição. Fonte: Autores.

Na Tabela 6 são apresentadas as médias dos erros referentes à propriocepção em $60^{\circ}$ e $30^{\circ}$ de extensão do joelho avaliadas pela dinamometria isocinética do participante 2 , com o percentual de diferença entre a avaliação basal vs $1^{\circ}$ dia; avaliação basal vs $5^{\circ}$ dia e avaliação $1^{\circ}$ dia vs $5^{\circ}$ dia. As médias das três tentativas seguidas mostram que houve melhora do senso de posição articular do joelho comparando os períodos após um dia de tratamento e após as cinco sessões para o ângulo de $60^{\circ}$ e $30^{\circ}$ de extensão, tendo em vista a redução dos erros. 
Tabela 6 - Médias dos erros nas três avaliações da propriocepção do participante 2 , nas angulações de $60^{\circ}$ e $30^{\circ}$ de extensão de joelho no dinamômetro isocinético.

\begin{tabular}{|c|c|c|c|c|c|c|c|c|}
\hline \multirow[b]{2}{*}{ Avaliações } & \multicolumn{2}{|c|}{ Angulações } & \multicolumn{3}{|c|}{$60^{\circ}$} & \multicolumn{3}{|c|}{$30^{\circ}$} \\
\hline & $60^{\circ}$ & $30^{\circ}$ & $\begin{array}{c}\% \text { Basal } \\
\text { vs } \\
1^{\circ} \text { dia }\end{array}$ & $\begin{array}{c}\% \text { Basal } \\
\text { vs } \\
5^{\circ} \text { dia }\end{array}$ & $\begin{array}{c}\% 1^{\circ} \text { dia } \\
\text { vs } \\
5^{\circ} \text { dia }\end{array}$ & $\begin{array}{c}\% \text { Basal } \\
\text { vs } \\
1^{\circ} \text { dia }\end{array}$ & $\begin{array}{c}\% \text { Basal } \\
\text { vs } \\
5^{\circ} \text { dia }\end{array}$ & $\begin{array}{c}\% 1^{\circ} \text { dia vs } \\
5^{\circ} \text { dia }\end{array}$ \\
\hline $\begin{array}{c}\text { Avaliação } \\
\text { basal }\end{array}$ & 2,7 & 3,7 & $\uparrow 148,15$ & $\uparrow 48,15$ & $\downarrow 40,30$ & $\uparrow 35,14$ & $\uparrow 16,22$ & $\downarrow 14,00$ \\
\hline $\begin{array}{c}1^{\circ} \text { sessão de } \\
\text { TTO }\end{array}$ & 6,7 & 5 & - & - & - & - & - & - \\
\hline $\begin{array}{c}5^{\circ} \text { sessão de } \\
\text { TTO }\end{array}$ & 4 & 4,3 & - & - & - & - & - & - \\
\hline
\end{tabular}

$\mathrm{TTO}=$ Tratamento; \%= Porcentagem; $\uparrow=$ Aumento; $\downarrow$ Diminuição. Fonte: Autores

Paciente 3, gênero feminino, 65 anos, sedentária, hipertensa em uso de medicação para controle, histórico de mais de duas quedas, com massa corporal de $68 \mathrm{~kg}, 1,68 \mathrm{~m}$ de altura, IMC de 24,09 e membro inferior direito dominante. Após distribuição aleatória por sorteio, a paciente realizou o treinamento sensório-motor em superfície estável.

A pontuação (score) obtida pela participante 3 na EEB está representada na Tabela 7, com o cálculo do percentual de diferença entre a avaliação basal vs $1^{\circ}$ dia; avaliação basal vs $5^{\circ}$ dia e avaliação $1^{\circ}$ dia vs $5^{\circ}$ dia. Os resultados mostram que, houve melhora do desempenho na EEB, tendo em vista o aumento do score obtido pela participante após um dia de tratamento, que se manteve após os cinco dias de intervenção.

Tabela 7 - Comparação das pontuações obtidas pela participante 3 na EEB nas três avaliações.

\begin{tabular}{|c|c|c|c|c|}
\hline Avaliações & Score & $\%$ Basal vs $1^{\circ}$ dia & $\%$ Basal vs $5^{\circ}$ dia & $\% 1^{\circ}$ dia vs $5^{\circ}$ dia \\
\hline Avaliação basal & 46 & $\uparrow 4,35$ & $\uparrow 4,35$ & 0 \\
\hline $1^{\circ}$ sessão de TTO & 48 & - & - & - \\
\hline $5^{\circ}$ sessão de TTO & 48 & - & - & - \\
\hline
\end{tabular}

$\mathrm{TTO}=$ Tratamento; $\%=$ Porcentagem; $\uparrow=$ Aumento. Fonte: Autores.

Na Tabela 8, estão descritos os resultados referentes ao valor de RMS dos músculos avaliados pela EMGS da participante 3 , com o percentual de diferença entre a avaliação basal vs $1^{\circ}$ dia; avaliação basal vs $5^{\circ}$ dia e avaliação $1^{\circ}$ dia vs $5^{\circ}$ dia. Observou-se que houve redução do RMS comparando as três avaliações dos músculos fibular longo e gastrocnêmio medial. O músculo vasto medial se destacou ao apresentar aumento da atividade elétrica comparando as três avaliações. Quanto ao glúteo médio, verificou-se redução da atividade elétrica após um dia de tratamento e aumento após os cinco dias de intervenção. 
Tabela 8 - Análise da EMGS dos músculos fibular longo, gastrocnêmio medial, vasto medial e glúteo médio da participante 3.

\begin{tabular}{ccccccc}
\hline $\begin{array}{c}\text { Músculo Valor } \\
\text { RMS (V) }\end{array}$ & $\begin{array}{c}\text { Avaliação } \\
\text { basal }\end{array}$ & $\begin{array}{c}\mathbf{1}^{\mathbf{o}} \text { sessão de } \\
\text { TTO }\end{array}$ & $\begin{array}{c}\mathbf{5}^{\mathbf{0}} \text { sessão de } \\
\text { TTO }\end{array}$ & $\begin{array}{c}\text { \% Basal } \\
\text { vs } \\
\mathbf{1}^{\mathbf{0}} \text { dia }\end{array}$ & $\begin{array}{c}\text { \% Basal } \\
\text { vs }\end{array}$ & $\begin{array}{c}\text { \% } \mathbf{1}^{\mathbf{0}} \text { dia } \\
\text { vs } \\
\mathbf{5}^{\mathbf{0}} \text { dia }\end{array}$ \\
\hline $\begin{array}{c}\text { Fibular longo } \\
\text { Gastrocnêmio } \\
\text { medial }\end{array}$ & 223,23 & 221,62 & 221,53 & $\downarrow 0,72$ & $\downarrow 0,76$ & $\downarrow 0,04$ \\
Vasto medial & 18,04 & 13,94 & 10,59 & $\downarrow 31,67$ & $\downarrow 48,09$ & $\downarrow 24,03$ \\
Glúteo médio & 16,21 & 14,25 & 21,03 & $\uparrow 3,44$ & $\uparrow 16,57$ & $\downarrow 12,70$ \\
\hline
\end{tabular}

$\mathrm{V}=$ Volt; TTO $=$ Tratamento; \%= Porcentagem; $\uparrow=$ Aumento; $\downarrow=$ Diminuição. Fonte: Autores.

Na Tabela 9 são apresentadas as médias dos erros referentes à propriocepção em $60^{\circ}$ e $30^{\circ}$ de extensão do joelho avaliadas pela dinamometria isocinética da participante 3 , com o percentual de diferença entre a avaliação basal vs $1^{\circ}$ dia; avaliação basal vs $5^{\circ}$ dia e avaliação $1^{\circ}$ dia vs $5^{\circ}$ dia. As médias das três tentativas seguidas mostram que, para o ângulo de $60^{\circ}$ e $30^{\circ}$ de extensão do joelho houve melhora do senso de posição articular, tendo em vista a redução dos erros ao comparar as três avaliações.

Tabela 9 - Médias dos erros nas três avaliações da propriocepção da participante 3, nas angulações de $60^{\circ}$ e $30^{\circ}$ de extensão de joelho no dinamômetro isocinético.

\begin{tabular}{|c|c|c|c|c|c|c|c|c|c|}
\hline \multirow[b]{2}{*}{ Avaliações } & \multicolumn{2}{|c|}{ Angulações } & \multicolumn{3}{|c|}{$60^{\circ}$} & \multicolumn{4}{|c|}{$30^{\circ}$} \\
\hline & $60^{\circ}$ & $30^{\circ}$ & $\begin{array}{c}\text { \% Basal } \\
\text { vs } \\
1^{\circ} \text { dia }\end{array}$ & $\begin{array}{c}\text { \% Basal } \\
\text { vs } \\
5^{\circ} \text { dia }\end{array}$ & $\begin{array}{c}\% 1^{\circ} \text { dia } \\
\text { vs } \\
5^{\circ} \text { dia }\end{array}$ & $\begin{array}{c}\text { \% Basal } \\
\text { vs } \\
1^{\circ} \text { dia }\end{array}$ & $\begin{array}{l}\% \text { Basal } \\
5^{0} \text { dia }\end{array}$ & vs & $\begin{array}{c}\% 1^{\circ} \text { dia } \\
\text { vs } \\
5^{\circ} \text { dia } \\
\end{array}$ \\
\hline Avaliação basal & 9 & 12,3 & $\downarrow 41,11$ & $\downarrow 52,22$ & $\downarrow 18,87$ & $\downarrow 37,40$ & $\downarrow 59,35$ & & $\downarrow 35,06$ \\
\hline $\begin{array}{c}1^{\text {o }} \text { sessão de } \\
\text { TTO }\end{array}$ & 5,3 & 7,7 & - & - & - & - & - & & - \\
\hline $\begin{array}{c}5^{\circ} \text { sessão de } \\
\text { TTO }\end{array}$ & 4,3 & 5 & - & - & - & - & - & & - \\
\hline
\end{tabular}

$\mathrm{TTO}=$ Tratamento; $\%=$ Porcentagem; $\uparrow=$ Aumento; $\downarrow$ Diminuição. Fonte: Autores.

\section{Discussão}

$\mathrm{Na}$ literatura atual encontram-se vários estudos sobre os benefícios da VCI para a população idosa, porém o protocolo ideal e a relação dose-resposta ainda não foram bem estabelecidos devido à variedade de parâmetros adotados nos diferentes estudos (Orr, 2015; Bacha et al., 2016; Pessoa et al., 2017; Rogan et al., 2017; Fischer et al., 2019). Alguns destes estudos apresentam limitações metodológicas, que comprometem a qualidade das pesquisas sobre o tema. Em um estudo realizado por Bigliassi et al. (2012) com 15 idosas, foi observado que o exercício agudo na plataforma vibratória não afetou o equilíbrio estático e dinâmico das voluntárias. Entretanto, os autores consideraram que o estudo apresentou limitações, como a falta de grupo controle para comparação intergrupos.

Para Rogan et al. (2017), a tomada de decisão no cuidado de idosos é uma tarefa complexa e deve ser baseada no melhor nível disponível de evidência científica com base em estudos bem desenhados, com rigor metodológico, que minimiza as chances de viés e que permite uma interpretação correta dos resultados. Em uma revisão de literatura realizada por estes mesmos autores, os protocolos dos estudos analisados diferiram significativamente entre eles, além de apresentarem pequenos números de amostras e muitos não terem realizado randomização cega. 
Em outra revisão de literatura realizada por Santos, França e Artigas (2019) sobre os benefícios da plataforma vibratória para a população idosa, também verificou-se que as abordagens metodológicas variam em relação aos protocolos utilizados. As frequências variaram entre 20 a $40 \mathrm{~Hz}$ e quanto ao período de treinamento, os autores observaram que este oscilava entre uma única sessão até 13 semanas de tratamento, sendo obtidos como principais desfechos a melhora do equilíbrio, ganho de força, melhora da funcionalidade e qualidade de vida.

No presente estudo, os resultados apontam que a participante 1 obteve melhora do equilíbrio corporal após somente um dia de VCI e que o tratamento continuou mostrando-se benéfico após os cinco dias de intervenção, havendo novamente um aumento no score na EEB. Um estudo realizado por Tsuji et al. (2014), constituído por 18 idosos com média de idade de 69,1 anos, apontou melhora no controle postural por meio do Teste de Alcance Funcional, após uma única sessão de VCI em plataforma com freqüência de $40 \mathrm{~Hz}$ e amplitude de $2-4 \mathrm{~mm}$.

As tarefas avaliadas pela EEB representam atividades simples da vida diária e seu uso foi capaz de demonstrar a melhora do equilíbrio da participante 1. De acordo com Silva \& Schneider (2011), um aspecto relevante que se deve levar em consideração ao pesquisar os efeitos da VCI no equilíbrio de idosos, é a evidência sobre a validade e a confiabilidade dos instrumentos empregados na medida dos desfechos.

Quanto a atividade elétrica muscular, os dados da participante 1 mostram que houve aumento do RMS de todos os músculos do membro inferior não dominante, avaliados neste estudo. De acordo com Bernardo-Filho et al. (2018), a VCI provoca uma contração muscular isométrica, conduz respostas adaptativas à exposição da vibração e oscilação de contrações musculares, alcançando muitos benefícios, dentre eles a melhora do equilíbrio postural, corroborando com os resultados do presente estudo.

Estudos que avaliaram a atividade eletromiográfica de músculos importantes na estabilização do tornozelo, como o fibular longo, durante diferentes exercícios proprioceptivos, mostraram a importância desses músculos na estabilização da articulação do tornozelo (Ferreira et al., 2009; Oliveira et al., 2012). Cunha e Bonfim (2007) realizaram um trabalho com oito mulheres para investigar a atividade elétrica dos músculos tibial anterior e fibular longo durante a manutenção da postura sobre uma prancha proprioceptiva em apoio unipodal e bipodal. Os resultados obtidos demonstraram que houve maior ativação do músculo fibular longo apenas nos exercícios em apoio unipodal na direção médio-lateral. Durante a VCI, sobretudo quando realizada em apoio unipodal, há uma perturbação capaz de gerar instabilidade médio-lateral no membro inferior, talvez isso explique o porquê deste músculo ter sido o mais ativado durante a terapia vibratória.

Goudarzian et al. (2017) acreditam que a vibração possa atuar como um estímulo mecânico, capaz de aumentar a atividade muscular, ativar os receptores articulares e gerar maior potencial nas propriedades neuromusculares. Essas adaptações neurais nos membros inferiores desempenham um papel importante no controle postural. Em um estudo realizado pelos autores com 22 idosas na faixa etária entre 60 e 80 anos, houve aumento da força muscular dos membros inferiores das voluntárias, além de melhora do equilíbrio dinâmico após dez dias consecutivos de VCI.

Fatores como a posição adotada durante o treinamento sobre a plataforma vibratória, também parecem ser relevantes. Na literatura encontram-se indicações de posturas nas mais variadas formas. Em um estudo realizado por Lam et al. (2016), uma única sessão de VCI proporcionou aumento da atividade muscular dos principais grupos musculares dos membros inferiores de idosos, sendo que este resultado foi mais significativo quando o treinamento foi executado na posição ereta em apoio unipodal. Estes mesmos autores ainda destacam que as diferenças no posicionamento alteram o grupo muscular que será mais ativado durante o exercício, assim como o recrutamento das fibras musculares.

O processo de envelhecimento promove uma alteração na velocidade das respostas musculares diante das perturbações no equilíbrio corporal. O treinamento proprioceptivo em plataformas instáveis é um importante recurso para a melhora do condicionamento neuromuscular. Durante o treinamento, a instabilidade dos movimentos exige mais das 
articulações, assim, a ativação dos impulsos proprioceptivos regula automaticamente os ajustes na contração muscular, reabilitando o equilíbrio (Gusmão \& Reis, 2017).

Em uma revisão recente da literatura realizada por Alam et al. (2018) sobre a VCI como um método de treinamento neuromuscular, concluiu que ela pode promover a melhora da força muscular, potência e flexibilidade, no entanto, os autores destacam que estes bons resultados não dependem apenas do protocolo adotado no treinamento, mas também da característica dos indivíduos tratados. Embora seja sedentária, a participante submetida à VCI no presente estudo, apresenta outras características que podem ter contribuído para sua boa evolução durante a pesquisa, como a ausência de doenças crônicas e de histórico de quedas e IMC considerado normal para o gênero e idade $(22,03)$.

Em relação à propriocepção, a participante 1 também obteve bons resultados através da VCI. Para o ângulo de $60^{\circ}$ de extensão do joelho, houve diminuição dos erros nas tentativas de percepção do senso de posição articular após os cinco dias de intervenção. Para o ângulo de $30^{\circ}$, a redução dos erros ocorreu após um dia de tratamento e após as cinco sessões. Vários aspectos do controle motor têm sido investigados observando o controle postural. Isso porque o controle postural é mantido por um sistema que sofre a ação de forças em constante mudança, sendo, portanto, razoável a sugestão de que esta orientação corporal é alcançada a partir de um relacionamento entre informação sensorial e ação motora. Neste caso, informação sensorial influencia a realização das ações motoras relacionadas ao controle postural e, simultaneamente, a realização destas ações motoras influencia a obtenção de informação sensorial (Ferling et al., 2019).

Sabe-se que o equilíbrio postural envolve tanto a capacidade de se recuperar de uma instabilidade, como a habilidade de antecipar o movimento para evitá-la. Logo, quando essas perturbações acontecem, são utilizadas estratégias de movimento para recuperar a estabilidade em resposta ao deslocamento do centro de gravidade. A articulação do joelho, escolhida para ser avaliada no atual estudo, contribui para essas estratégias, porém, é uma das articulações mais acometidas por alterações crônico-degenerativas nos idosos. Tais alterações podem causar instabilidade articular e prejuízos na propriocepção, que alteram o controle postural. (Souza et al. 2017).

Os resultados dos três participantes mostram que houve melhora do equilíbrio postural, da atividade elétrica dos músculos avaliados e redução de erros nas tentativas de percepção do senso de posição articular do joelho. Portanto, os dados obtidos neste estudo mostram que, ambos os tratamentos foram benéficos para os idosos, porém a participante que realizou o exercício unipodal associado à VCI obteve melhores resultados. Acredita-se que o sistema proprioceptivo pode condicionar-se com exercícios específicos e que a VCI possa atuar de forma complementar aos estímulos que já são fornecidos em superfície estável.

Cabe ressaltar que o participante 2 é sedentário, diabético em uso de medicação para controle e possui um IMC considerado acima do ideal para o gênero e idade $(27,68)$. A participante 3 também é sedentária, hipertensa em uso de medicação para controle, tem histórico de mais de duas quedas e apresentou um desempenho muito baixo na EEB. Estes fatores podem ter contribuído para resultados menos expressivos em alguns aspectos avaliados no estudo. Doenças crônicas como o Diabetes Mellitus podem aumentar o déficit de equilíbrio e consequentemente o risco de quedas na população idosa, devido a fatores como desenvolvimento de neuropatia, retinopatia, alteração da glicemia e/ou polifarmácia, sendo este último um fator também relacionado à Hipertensão Arterial Sistêmica. O sobrepeso pode estar associado às doenças crônicas e também contribui para maior prevalência de quedas entre os idosos (Fontes et al., 2019; Rodrigues et al., 2020).

O projeto deste trabalho tinha como propósito realizar um estudo clínico randomizado sobre o tema, porém não foi possível completar a amostra desejada devido à pandemia da COVID-19. Optou-se pela interrupção da coleta de dados, já que os voluntários do estudo fazem parte do grupo de risco. Apesar disso, este estudo apresenta uma importante implicação clínica e pode ser útil para a elaboração de novas pesquisas sobre o assunto. 


\section{Conclusão}

À medida que se conhece mais sobre os efeitos da terapia vibratória, é possível utilizá-la na área da engenharia biomédica para beneficiar pacientes com diferentes necessidades. Neste estudo, verificou-se que ambos os tratamentos foram benéficos para os participantes.

A idosa que realizou o exercício unipodal associado à VCI obteve melhora no equilíbrio postural, tendo em vista o aumento do score da EEB nas avaliações após um dia de VCI e após o fim do tratamento. Quanto à análise muscular, em todos os músculos avaliados houve aumento da atividade elétrica nos períodos de avaliação. Com relação à propriocepção, também foram verificados alguns benefícios, sendo obtida redução dos erros de percepção do senso de posição articular do joelho em algumas das angulações testadas.

Os idosos que realizaram o treinamento sensório-motor no solo apresentaram resultados semelhantes. Houve melhora no equilíbrio postural, sendo observado aumento do score da EEB após um dia de tratamento, que se manteve após os cinco dias de intervenção. Com relação à avaliação muscular, alguns músculos se destacaram apresentando aumento da atividade elétrica. Quanto à propriocepção, também foi verificada a redução dos erros de percepção do senso de posição articular do joelho em algumas das angulações testadas.

Estes achados são promissores para o uso do treinamento sensório-motor com a população idosa, porém sugere-se que sejam realizados estudos clínicos, capazes de elucidar sobre protocolos ideais de VCI a serem utilizados nesta faixa etária.

\section{Referências}

Agudelo-Botero, M., Giraldo-Rodríguez, L., Murillo-González, J. C., Mino-León, D. \& Cruz-Arenas, E. (2018). Factors associated with occasional and recurrent falls in Mexican community-dwelling older people: PLOS ONE, 13(2), 1-12.

Alam, M. M., Khan, A. A. \& Farooq, M (2018). Effect of whole-body vibration on neuromuscular performance: A literature review. Work, 59(4), 571-83.

Amaral, P. C (2012). Plataforma Vibratória: Introdução ao treinamento vibratório: Livro didático. Editora Paulo Amaral.

Bacha, J. M. R., Cordeiro, L. R., Alvisi, T. C. \& Bonfim, T. R. (2016). O impacto do treinamento sensório-motor com plataforma vibratória no equilíbrio e na mobilidade funcional no indivíduo idoso com sequela de acidente vascular encefálico: relato de caso. Fisioterapia e Pesquisa, 23(1), 111-6.

Batista, M. A. B., Wallerstein, L. F., Dias, R. M., Silva, R. G., Ugrinowitsch, C., Tricoli, V. (2007). Efeitos do Treinamento com Plataformas Vibratórias. Revista Brasileira de Ciência \& Movimento, 15(3), 103-113.

Berg, K. O., Wood-Dauphinée, S., Williams, J., Maki, B. (1992). Measuring balance in the elderly: validation of an instrument. Canadian Journal of Public Health, 83(2), 7-11.

Bernardo-Filho, M., Bemben, D., Stark, C. \& Taiar, R. (2018). Biological consequences of exposure to mechanical vibration. Dose Response, 16(3), 1559325818799618.

Bigliassi, M., Costa, E. G. F., Souza, F. F., Teixeira, D. C., Júnior, R. A. S., Júnior, R. P. \& Altimari, L. R. (2012). Efeito do exercício agudo realizado na plataforma vibratória sobre o equilíbrio estático e dinâmico de idosos. Geriatrics, Gerontology and Aging, 6(3), $254-260$.

Cardinale, M. \& Lim, J (2003). Electromyography activity of vastus lateralis muscle during whole-body vibrations of different frequencies. The Journal of Strength \& Conditioning Research, 17(3), 621-4.

Claudino, R., Roecker, R., Azevedo, A. K. C. \& Jackson, S. G. (2015). Análise das estratégias de ajustes posturais do glúteo médio na presença de perturbações em idosos e jovens. Arquivos de Ciências da Saúde, 22(2), 63-68.

Costa, J. S. \& Navarro, D. N. (2016). Exercício Fìsico e Sarcopenia. Revista Brasileira de Prescrição e Fisiologia do Exercício, $10(58), 209-213$.

Cruz-Jentoft, A. J., Bahat, G., Bauer, J. M. \& Boirie, Y. (2019). Sarcopenia: revised European consensus on definition and diagnosis. Age Ageing, 48(1), 1631.

Cunha, P. L. \& Bonfim, T. R. (2007). Ativação eletromiográfica em exercícios sobre a prancha de equilíbrio. Fisioterapia Brasil, 8(3), $192-7$.

Costa, J. P., Vitorino, R., Silva, G. M., Vogel, C., Duarte, A. C. \& Rocha-Santos, T. (2016). A synopsis on aging-Theories, mechanisms and future prospects. Ageing Research Reviews, 29, p. 90-112, 2016.

Dantas, E. H. M. \& Santos, C. A. S. (2017). Aspectos biopsicossociais do envelhecimento e a prevenção de quedas na terceira idade. Joaçaba: Editora Unoesc. 
Diego, I. M. A., Hernández, C. P., Rueda, F. M., Cuerda, R. C. (2012). Efectos de lavibroterapia sobre elcontrol postural, lafuncionalidad y la fatiga en pacientes conesclerosis múltiple. Ensayo clínico aleatorizado. Neurología, 27(3), 143-153.

Diz, J. B. M., Leopoldino, A. A. O., Moreira, B. S., Henschke, N., Dias, R. C., Pereira, L. S. M. \& Oliveira, V. C. (2017). Prevalence of sarcopenia in older Brazilians: A systematic review and meta-analysis. Geriatrics and Gerontology International, 17(1), 5-16.

Donath, L. (2015). Different ankle muscle coordination patterns and co-activation during quiet stance between young adults and seniors do not change after a bout of high intensity training. BMC Geriatrics, 15(19), 1-8.

Ferlinc, A., Fabiani, E., Velnar, T. \& Gradisnik, L. (2019). The importance and role of proprioception in the elderly: a short review. Materia Socio-Medica, $31(3), 219-221$

Ferreira, L. A. B., Rossi, L. P., Pereira, W. M., Vieira, F. F. \& Júnior, A. R. P. (2009). Análise da atividade eletromiográfica dos músculos do tornozelo em solo estável e instável. Fisioterapia em Movimento, 22(2), 177-187.

Fischer, M., Vialleron, T., Laffaye, G., Fourcade, P., Hussein, T., Chèze, L., Deleu, P. A., Honeine, J. L., Yiou, E. \& Delafontaine, A. (2019). Long-term effects of whole-body vibration on human gait: a systematic review and meta-analysis. Frontiers in Neurology, 10(627), 1-24.

Flores, B. C., Wagner, L. E., Hopp, M. S., Renner, J. D. P., Cardoso, D. M., Machado, G. R. \& Paiva, D. N. (2018). Efeitos do treino com plataforma vibratória sobre a força muscular e capacidade funcional em idosas hígidas. Saúde (Santa Maria), 44(3), 1-11.

Fontes, F. P., Correia, L. C., Lima Filho, B. F. \& Gazzola, J. M. (2019). Intervenção fisioterapêutica na melhora do equilíbrio em idoso com diabetes mellitus tipo 2: uma revisão sistemática. Revista Brasileira de Ciência e Movimento, 27(3), 200-209.

Freeman, M. A. R. (1965). Instabilidade do pé após lesões o ligamento lateral do tornozelo. The Journal of Bone and Joint Surgery, 47(4), 669-6777.

Goudarzian, M., Ghavi, S., Shariat, A., Shirvani, H. \& Rahimi, M. (2017). Effects of whole body vibration training and mental training on mobility, neuromuscular performance, and muscle strength in older men. Journal of Exercise Rehabilitation, 13(5), 573-580.

Gusmão, M. F. S. \& Reis, L. A. (2017). Efeitos do treinamento sensório-motor no equilíbrio de idosos: revisão sistemática. Revista de Saúde Coletiva da Universidade Estadual de Feira de Santana, 7(1), 64-70.

Hayter, A. K. M., Jeffery, R., Sharma, C., Prost, A. \& Kinra, S. (2015). Community perceptions of health and chronic disease in South Indian rural transitional communities: a qualitative study. Global Health Action, 8(1), 1-8.

Hunter, G. R., Mccarthy, J. P., Bamman, M. M. (2004). Effects of resistance training on older adults. Sports Medicine, 34(5), 330-348.

Instituto Brasileiro de Geografia e Estatística (IBGE (2019)). Tábua completa de mortalidade para o Brasil - 2019. Breve análise da evolução da mortalidade no Brasil.

Instituto Brasileiro de Geografia e Estatística (IBGE) - Diretoria de Pesquisas, Coordenação de Trabalho e Rendimento - Pesquisa Nacional por Amostra de Domicílios Contínua (2012/2019). Conheça o Brasil - População Pirâmide Etária. <https://educa.ibge.gov.br/jovens/conheca-o-brasil/populacao/18318piramide etaria.html\#: :text=Os\%20grupos\%20de\%2030\%20a,10\%2C8\%25\%20da\%20populp\%C3\%A7\%C3\%A3o>

Jacob Filho, W., Jorge, A. A. L., Busse, A. L., Galvão, C. E. S., Silva, F. P., Tibério, I. F. L. C., Andrade, L. C., Drager, L. F., Machado, M, C., Olmos, R. D., Pereira, R. M. R., Gualandro, S. F. M. (2015). Envelhecimento Uma Visão Interdisciplinar. Editora Atheneu.

Janda, V. (2000). Manuelle Muskelfunktions-diagnostik. Amsterdã: Elsevier.

Júnior, B. A. L., Oliveira, C. A. S., Bezerra, E. L., Santana, R. P., Cerdeira, D. Q. (2019). Caracterização dos principais exercícios terapêuticos na diminuição de quedas em idosos: revisão integrativa. Brazilian Journal of Health Review, 2(4), 2365-2375.

Kilby, M. C. (2015). Models of postural control: shared variance in joint and COM motions. Plos One, 10(5), 1-10.

Lam, F. M.H. (2016). The effect of vertical whole-body vibration on lower limb muscle activation in elderly adults: Influence of vibration frequency, amplitude and exercise. Maturitas, 88, 59-64.

Lamb, M. 1. (2014). Efeito do treinamento proprioceptivo no equilíbrio de atletas de ginástica rítmica. Revista Brasileira de Medicina do Esporte, 20(5), 379382 .

Locquet, M. (2019). Association between the decline in muscle health and the decline in bone health in older individuals from the sarcophage cohort. Calcified Tissue International, 104(3), 273- 284.

Margutti, K. M. M., Schuch, N. J. \& Schwanke, C. H. A. (2017). Inflammatory markers, sarcopenia and its diagnostic criteria among the elderly: a systematic review. Revista Brasileira de Geriatria e Gerontologia, 20(3), 441-453.

Matsumoto, H., Tanimura, C., Tanishima, S., Osaki, M., Noma, H. \& Hagino, H. (2017). Sarcopenia is a risk factor for falling in independently living Japanese older adults: a 2-year prospective cohort study of the GAINA study. Geriatrics \& Gerontology International, 17(11), 2124-2130.

McGrath, T. M., Waddington, G., Scarvell, J. M., Ball, N. B., Creer, R., Woods, K. \& Smith, D. (2016). The effect of limb dominance on lower limb functional performance - A systematic review. Journal of Sports Sciences, 34(4), 289-302.

Mcphee, J. S., French, D. P., Jackson, D., Nazroo, J., Pendleton, N. \& Degens, H. (2016). Physical activity in older age: perspectives for healthy ageing and frailty. Biogerontology, 17(3), 567-80.

Mesquita, J. S., Cavalcante, M. R. L. \& Siqueira, C. A. (2016). Promoção da saúde e integralidade na atenção ao idoso: uma realidade brasileira? Revista Kairós Gerontologia,19(1), 227-38. 
Ministério da Saúde (2012). Resolução n 466, de 12 de dezembro de 2012. Diário Oficial da União, 13 de junho de 2013. Seção 1 , p. 59.

Miyamoto, S. T., Junior, I. L., Berg, K. O., Ramos, L. R. \& Natour, J. (2004). Brazilian version of the Berg balance scale. Brazilian Journal of Medical and Biological Research, 37(9),1411-1421.

Moore, K. L., Dalley, A. F. \& Agur, A. M. R. (2018). Anatomia Orientada para a Clínica. Editora Guanabara Koogan.

Morse, C. I., Thom, J. M., Birch, K. M. \& Narici, M. V. (2005). Changes in triceps surae muscle architecture with sarcopenia. Acta Physiologica Scandinavica, 183(3), 291-8.

Narici, M. V., Maganaris, C. N., Reeves, N. D. \& Capodaglio, P. (2003). Effect of aging on human muscle architecture. Journal of Applied Physiology, 95(6), 2229-2234.

Nascimento, M. M. (2020). Uma visão geral das teorias do envelhecimento humano. Revista Saúde e Desenvolvimento Humano, 8(1), $161-168$.

Neto, J. P., Brito, C. A. F. (2015). Mobilidade funcional em função da força muscular em mulheres idosas fisicamente ativas. Revista Brasileira de Medicina do Esporte, 21(5), 369-175.

Oliveira, D. C. S., Rezende, P. A. M. S. L., Silva, M. R., Lizardo, F. B., Sousa, G. C., Santos, L. A., Guimarães, E. A. \& Chacur, E. P. (2012). Análise eletromiográfica de músculos de músculos do membro inferior em exercícios proprioceptivos realizados com olhos abertos e fechados. Revista Brasileira de Medicina do Esporte, 18(4), 261-266.

Organização Mundial da Saúde (2018). Quedas. https://www.who.int/en/news-room/fact-sheets/detail/falls>.

Organização Mundial da Saúde (OMS) (2020). Orientações sobre atividade física $e$ comportamento sedentário. <https://www.who.int/publications/i/item/9789240015128>.

Orr, R. (2015). The effect of whole body vibration exposure on balance and functional mobility in older adults: A systematic review and meta-analysis. Maturitas, 80(4),342-358.

Oroszi, T., Heuvelen, M. J. G., Nyakas, C. \& Zee, E. A. (2020). Vibration detection: its function and recent advances in medical applications. F1000Research, $17: 9,619$.

Paiva, D., Cabezuelo, P. J. M. \& Filho, M. B. (2014). Plataforma Oscilante/Vibratória: Fundamentos e a Prática Clínica. Editora Andreoli.

Park, S., Kong, Y. S., Ko, Y. M., Jang, G. U. \& Park, J. W. (2015). Differences in onset timing between the vastus medialis and lateralis during concentric knee contraction in individuals with genu varum or valgum. Journal of Physical Therapy Science, 27(4), 1207-1210.

Pessoa, M. F., Brandão, D. C., Sá, R. B., Souza, H. C. M., Fuzani, H. K. B. \& Andrade, A. D. (2017). Effects of Whole Body Vibration on muscle strength and quality of life in health elderly: A meta-analysis. Fisioterapia em Movimento, 30(1), 171-82.

Pimentel, W. R. T., Pagotto, V., Stopa, S. R., Hoffmann, M. C. C. L., Andrade, F. B., Junior, P. R. B. S., Lima-Costa, M. F. \& Menezes, R. L. (2018). Quedas entre idosos brasileiros residentes em áreas urbanas: ELSI-Brasil. Revista de Saúde Pública, 52(2), 1-9.

Queiroz, K. V., Barbosa, W. T., Cerdeira, D. Q., Barros, L. L. S. \& Arcanjo, G. N. (2018). Avaliação dos ísquios tibiais através do ângulo poplíteo em pacientes idosos com artrose de joelho. Fisioterapia Brasil, 20(6), 713-720.

Richardson, J. K. (2017). The confusing circular nature of falls research. and a possible antidote. American Journal of Physical Medicine \& Rehabilitation, 96(1), 55-59.

Rittweger, J. (2010). Vibration as an exercise modality: how it may work, and what its potential might be. European Journal off Applied Physiology, 108(5), $877-904$.

Rodrigues, E. H. P. \& Bertoncello, D. (2017) Análise eletromiográfica durante exercícios de propriocepção de tornozelo em apoio unipodal. ConScientiae Saúde, 16(1), 73-81.

Rodrigues, A. E. C., Taeymans, J., Radlinger, L., Naepflin, S., Ruppen, S., Bruelhart, Y. \& Hilfiker, R. (2020). Mulheres idosas obesas apresentam maior prevalência de quedas e pior equilíbrio estático e dinâmico? Um estudo transversal. Brazilian Journal of Development, 6(11), 89242-89254.

Rogan, S., Hilfiker, R., Herren, K., Radlinger, L. \& Bruin, E. D. (2017). Effects of whole-body vibration on postural control in elderly: An update of a systematic review and meta-analysis. Archives of Gerontology and Geriatrics, 73, 95-112.

Santos, K., Goedert, A., Bento, P. \& Rodacki, A. (2015). Associação entre exercício físico e propriocepção em idosos: uma revisão sistemática. Revista Brasileira de Atividade Física \& Saúde, 20(1), 17-25.

Santos, S. M., França, L. S. \& Artigas, N. R. (2019). Os benefícios da plataforma vibratória na melhora dos déficits neuromusculares do envelhecimento: uma revisão de literatura. Saúde e Desenvolvimento Humano,7(3), 73-80.

Schmidt, B. G., Gerzson, L. R. \& Almeida, C. S. (2020). O uso da eletromiografia de superfície como medida de desfecho da fisioterapia em crianças com Paralisia Cerebral: uma revisão sistemática. Journal of Human Growth and Development, 30(2), 216-226.

Scianni, A. A., Faria, G. S., Silva, J. S., Benfica, P. A. \& Faria, C. D. C. M. (2019). Effects of physical exercises on the nervous system of elders and its functional consequences. Revista Brasileira de Ciências do Esporte, 41(1), 81-95.

Silva, P. Z. \& Schneider, R. H. (2011). Efeitos da plataforma vibratória no equilíbrio em idosos. Acta Fisiátrica, 18(1), 21-26. 
Research, Society and Development, v. 10, n. 16, e543101624032, 2021

(CC BY 4.0) | ISSN 2525-3409 | DOI: http://dx.doi.org/10.33448/rsd-v10i16.24032

Souza, A. A., Moura, J. A. \& Bastone, A. C. (2017). Efetividade de um programa de fisioterapia aquática na capacidade aeróbia, dor, rigidez, equilíbrio e função física de idosos com osteoartrite de joelho. Fisioterapia Brasil, 18(2), 165-171.

Sumukadas, D., Price, R. J. G., McMurdo, M. E. T. \& Rauchhaus, P. (2018). The effect of perindopril on postural instability in older people with a history of falls-a randomised controlled trial. Age and Ageing, 47(1), 75-81

Surface Electromyography for the Non Invasive Assessment of Muscle (SENIAM). Recommendations for sensor locations on individual muscles. <http://www.seniam.org/>.

Takakusaki, K. (2017). Functional neuroanatomy for posture and gait control. Journal of Movement Disorders, 10(1), 1-17.

Thomaz, R. A. M (2015). Efeitos dos exercícios proprioceptivos associados a estímulos vibratórios na atividade eletromiográfica, oscilação postural e senso de posição articular em indivíduos saudáveis. 55 f. Dissertação (Mestrado em Bioengenharia) - Universidade do Vale do Paraíba, São José dos Campos - SP.

Tsuji, T., Kitano, N., Tsunoda, K., Himori, E., Okura, T. \& Tanaka, K. (2014). Short-term effects of Whole-Body Vibration on functional mobily and flexibility in healthy, older adults. Journal of Geriatric Physical Therapy, 37(2), 58-64.

Varella,, L. R. D., Torres, V. B., Angelo, P. H. M., Oliveira, M. C. E., Barros, A. C. M., Viana, E. S. R. \& Micussi, M. T. A. B. C. (2016). Influence of parity, type of delivery, and physical activity level on pelvic floor muscles in postmenopausal women. Journal of Physical Therapy Science, 28(3), 824-830.

Veras, R. (2016). É possível, no Brasil, envelhecer com saúde e qualidade de vida? Revista Brasileira de Geriatria e Gerontologia, 19(3), 381-382.

Veras, R. P. \& Oliveira, M. (2018). Envelhecer no Brasil: a construção de um modelo de cuidado. Revista Ciência \& Saúde Coletiva, 23(6), 1929-1936. 